\title{
Structure of flux line lattices with weak disorder at large length scales
}

\section{Citation}

Kim, Philip, Zhen Yao, Cristian A. Bolle, and Charles M. Lieber. 1999. "Structure of Flux Line Lattices with Weak Disorder at Large Length Scales." Physical Review B 60 (18): R12589-92. https://doi.org/10.1103/physrevb.60.r12589.

\section{Permanent link}

http://nrs.harvard.edu/urn-3:HUL.InstRepos:41417431

\section{Terms of Use}

This article was downloaded from Harvard University's DASH repository, and is made available under the terms and conditions applicable to Other Posted Material, as set forth at http:// nrs.harvard.edu/urn-3:HUL.InstRepos:dash.current.terms-of-use\#LAA

\section{Share Your Story}

The Harvard community has made this article openly available.

Please share how this access benefits you. Submit a story.

Accessibility 


\title{
Structure of Flux Line Lattices with Weak Disorder at Large Length Scales
}

\author{
Philip Kim, Zhen Yaot, Cristian A. Bolle \\ Division of Engineering and Applied Sciences, Harvard University, Cambridge, MA 02138
}

\begin{abstract}
Dislocation-free decoration images containing up to 80,000 vortices have been obtained on high quality $\mathrm{Bi}_{2} \mathrm{Sr}_{2} \mathrm{CaCu}_{2} \mathrm{O}_{8+x}$ superconducting single crystals. The observed flux line lattices are in the random manifold regime with a roughening exponent of 0.44 for length scales up to 80-100 lattice constants. At larger length scales, the data exhibit nonequilibrium features that persist for different cooling rates and field histories.
\end{abstract}

PACS numbers: 74.60.Ge, 75.10.Nr, 74.60.Ec, 74.72.Hs

Recent studies of high temperature superconductors have shown richness in the phase diagram due to the presence of weak quenched disorder [1]. Larkin first showed that arbitrarily weak disorder destroys the long range translational order of flux lines (FLs) in a lattice [2]. It was recently pointed out that the Larkin model, which is based on a small displacement expansion of the disorder potential, cannot be applied to length scales larger than the correlated volume of the impurity potential termed the Larkin regime [3 5]. Beyond the Larkin regime, the behavior of FLs in the absence of dislocations has been considered using elastic models 3 5]. First, FLs start to behave collectively as an elastic manifold in a random potential with many metastable states (the random manifold regime) [3]. In this random manifold regime, the translational order decreases as a stretched exponential, whereas there is a more rapid exponential decay in the Larkin regime. At even larger length scales, when the displacement correlation of FLs become comparable to the lattice spacing, the random manifold regime transits to a quasiordered regime where the translational order decays as a power law [4,5].

Experimentally, neutron diffraction $[6$ and local Hall probe measurements [7] have shown the existence of an order-disorder phase transition with increased field, although the microscopic details of these phases are not clear. Theoretical progress describing FLs in the presence of weak disorder has been made within elastic theory, which proposes the absence of dislocations at equilibrium [5,8,9]. To date, however, there has been no experimental work addressing the structure of dislocation-free FL lattices at large length scales. Previous magnetic decoration studies [10]11] showed that the dislocation density decreases and the translational order increases with increasing magnetic field. However, only relatively shortrange translational order could be probed in the previous work due to the finite image size and relatively low applied fields.

In this paper, we report the first large length scale structural studies of FLs with measurements extending up to $\sim 300$ lattice constants and fields up to $120 \mathrm{G}$. Realspace images show dislocation free regions containing up to the order of $10^{5}$ FLs. A very low density of dislocations was also observed, although detailed analysis suggests that the dislocations are not equilibrium features. The translational correlation function and displacement correlator have been calculated from dislocation free data to examine quantitatively the decay of order. These results show a stretched exponential decay of the translational order indicating that FLs are in the random manifold regime. The experimentally determined roughening exponent in the random manifold regime agrees well with theoretical predictions.

High quality single crystals of $\mathrm{Bi}_{2} \mathrm{Sr}_{2} \mathrm{CaCu}_{2} \mathrm{O}_{8+x}$ (BSCCO) were grown as described elsewhere 12. Typically, crystals of $\sim 1 \mathrm{~mm} \times 1 \mathrm{~mm} \times 20 \mu \mathrm{m}$ size were mounted on a copper cold-finger and decorated with thermally evaporated iron clusters at $4 \mathrm{~K}$. The samples were cooled down to $4 \mathrm{~K}$ using different thermal cycles to test nonequilibrium effects and to achieve as close an equilibrium configuration of FLs as possible within the experimental time scale. The FL structure was imaged after decoration using a scanning electron microscope equipped with a $4096 \times 4096$ pixel, 8-bit gray-scale image acquisition system. Nonlinearity in the system was eliminated using grating standards. This high-resolution system enabled us to acquire images containing nearly $10^{5} \mathrm{FLs}$, while maintaining a similar resolution $(\sim 14$ pixels between vortices) to previous studies of $10^{3} \mathrm{FLs}$. In addition, an iterative Voronoi construction [13] was used to reduce the positioning inaccuracy to $3 \%$ of a lattice constant.

Samples were decorated in fields of 70,80 and 120 $\mathrm{G}$ parallel to the c axis of BSCCO single crystals. In contrast to the previous decoration experiments at lower fields [10,11], we find that the dislocations are rare at these fields. The density of dislocations was $1.7 \times 10^{-5}$, $1.4 \times 10^{-5}$ and $3.1 \times 10^{-5}$ for 70,80 and $120 \mathrm{G}$, respectively, where the total number of vortices is $\sim 240,000$ for each field. It is thus trivial to find many large $100 \times 100$ $\mu \mathrm{m}^{2}$ dislocation free regions in the decorated samples. The size of the largest dislocation free image, which was obtained in a field of $70 \mathrm{G}$, is $152 \times 152 \mu \mathrm{m}^{2}$ with 78,363 vortices 14]. Although a small number of dislocations are detected in our FL images, this does not imply that they are energetically favorable at equilibrium. On the 
contrary, we believe that the large dislocation-free areas observed in the images provide a lower bound for the length scale of equilibrium dislocation loops. We discuss this point below after presenting a quantitative analysis of the translational order.

To study quantitatively the FL lattice order, we proceed as follows. First, a perfect lattice is constructed and registered to the FL positions obtained from an experimental image. The initial lattice vectors used to construct the perfect lattice were obtained from the Fourier transform of the vortex positions. When an image contains a dislocation, the continuum approximation is used to construct the perfect lattice with the dislocation [15]. Next we minimized the root mean square displacement between the underlying perfect lattice and the real FL lattice by varying the position and orientation of the two lattice vectors of the perfect lattice. The displacement vector $\mathbf{u}(\mathbf{r})$ associated with each of the vortices positioned at $\mathbf{r}$ relative to the perfect lattice was then computed. Fig 1 displays a color-representation of the displacement field for a typical dislocation-free image and an image containing three dislocations. In Fig 1(a), the average displacement is $0.22 a_{0}$, where $a_{0}$ is the lattice constant. Qualitatively, the map consists of several intermixed domain-like structures, within which the displacement fields are correlated. These uniformly dispersed domain-like structures of the displacement field produce sharp Bragg peaks in Fourier space (see Fig $3(\mathrm{~b})$ later). We also believe that $\mathbf{u}(\mathbf{r})$ provides a quick indication of nonequilibrium effects. For example, Fig 1 (b) exhibits large domains of correlated displacements that are sheared relative to each other; that is, the blue-greenblue coded domains. We believe that this larger scale distortion is a manifestation of a nonequilibrium structure that may arise from quenched dynamics of FLs during our field-cooling process (see below).

To compare our data directly with theoretical predictions [5], we have calculated the displacement correlator, $B(r)$, and translational correlation function, $C_{\mathbf{G}}(r)$. $B(r)$ and $C_{\mathbf{G}}(r)$ are defined as $\left\langle[\mathbf{u}(\mathbf{r})-\mathbf{u}(\mathbf{0})]^{2}\right\rangle / 2$ and $\left\langle e^{i \mathbf{G}[\mathbf{u}(\mathbf{r})-\mathbf{u}(\mathbf{0})]}\right\rangle$, respectively, where \langle\rangle is the average over thermal fluctuations and quenched disorder, and $\mathbf{G}$ is one of the reciprocal lattice vectors. Theoretically [5], we expect $B(r)$ will show three distinct behaviors as $r$ increases: $B(r) \sim r$ in the Larkin regime, where $B(r)$ is less than the square of $\xi$, the in-plane coherence length. As $r$ increases further, FLs are in the random manifold regime where $\xi^{2}<B(r)<a_{0}^{2}$. In this regime $B(r) \sim r^{2 \nu}$ with the roughening exponent $2 \nu(<1)$. Finally, at the largest length scales (the quasiordered regime) where $a_{0}^{2}<B(r)$, $B(r) \sim \ln r$. Since the in-plane $\xi$ of BSCCO is only $\sim 20 \AA$, the Larkin regime is irrelevant in our experiment (i.e., $a_{0} \gg \xi$ ). Fig 2(a) shows the behavior of $\mathrm{B}(\mathrm{r})$ calculated from the data in Fig 1 (a). For $r<80 a_{0}$, $B(r)$ can be fit well with a power law, $B(r) \sim r^{2 \nu}$, with $2 \nu=0.44$. Thus our experiment is probing the ran- dom manifold regime at least up to this scale. Indeed, $B(r)$ grows only up to $0.05 a_{0}^{2}$ at $r=80 a_{0}$, well below the expected crossover to the quasiordered regime, i.e. $B(r) \sim a_{0}^{2}$. A naive extrapolation to $B(r)=a_{0}^{2}$ suggests the crossover at $r \sim 10,000 a_{0}(\sim 4 \mathrm{~mm})$, which is far beyond our experimental limit. Samples with such a large clean area, and direct imaging of $\sim 10^{8}$ vortices would be required to observe the logarithmic roughening of FLs. The roughening exponent $2 \nu$ is found to be independent of the field $(70-120 \mathrm{G})$ and consistent with the estimate $2 \nu=2 / 5$ obtained by Feigelman et al. using a scaling argument [3]. As shown in Fig 2(b), $C_{\mathbf{G}}(r)$ and $e^{-G^{2} B(r) / 2}$ overlap with each other for $r<L^{*}$, where the measured $L^{*}$ is $\sim 80 a_{0}$. These results support the Gaussian approximation, $C_{\mathbf{G}}(r) \approx e^{-G^{2} B(r) / 2}$, which has been simply assumed for the equilibrium FLs lattice [5] within this length scale. For $r>L^{*}$, however, B(r) deviates strongly from expected behavior; that is, $B(r)$ saturates and even decreases as $r$ increases. In addition, the Gaussian approximation breaks down for $r>L^{*}$ as evidenced by the difference between $C_{\mathbf{G}}(r)$ and $e^{-G^{2} B(r) / 2}$. We believe that this behavior can be attributed to nonequilibrium FL structures at the larger length scales of our experiment.

To examine this point further, we decompose $B(r)$ into its longitudinal $\left[B^{L}(r)\right]$ and transverse $\left[B^{T}(r)\right]$ parts: $B(r)=\left(B^{L}(r)+B^{T}(r)\right) / 2$, where

$$
B^{L}(r)=\left\langle\left((\mathbf{u}(\mathbf{r})-\mathbf{u}(\mathbf{0})) \cdot \frac{\mathbf{r}}{r}\right)^{2}\right\rangle .
$$

It is worth noting that in the random manifold regime, the ratio of $B^{T}(r)$ and $B^{L}(r)$ is predicted to be $2 \nu+1$ [5], and thus an independent estimate of the roughening exponent. The average value of this ratio measured from our data (inset to Fig B(a)) is 1.40, which is consistent with the value of $2 \nu$ obtained from $B(r)$. As shown in Fig 3(a), both $B^{L}$ and $B^{T}(r)$ are described well with the power law behavior up to $r \sim L^{*}$. Beyond this range, however, the transverse displacement $B^{T}(r)$ first deviates from power law causing deviations in $B(r)$. Thus, we infer that shear motion of FL lattice should be responsible for the abnormal behavior of $B(r)$. Since the shear modulus of FL lattice is much smaller in magnitude than the compressional modulus [1], $B^{T}(r)$ is always larger than $B^{L}(r)$, and the shear motion dominates the relaxation of the FL lattice during the field cooling process. As temperature decreases, the long wavelength component of shear motion is frozen out. We believe that the domain-like structures seen in Fig 1 are a snap shot of these frozen long wavelength shear motions. Note that the characteristic length scale of these domain like structures in Fig 1 is again $\sim L^{*}$, which explains the deviations in $B(r)$ for $r>L^{*}$. Therefore, $L^{*}$ is the equilibrium length scale within which FLs can relax to the local equilibrium during our experimental time scale. 
This issue can also be addressed through Fourier space analysis. Fig 3(b) displays a blow-up of one Bragg peak. Several small satellite peaks appear around the relatively sharp main peak; these satellite peaks indicate a largescale modulation of the FL lattice. If the FLs were in equilibrium, only one main peak should be expected. The corresponding real space distance between the main and satellite peaks is, again, $\sim L^{*}$. Hence these satellite peaks provide another evidence of the frozen-in dynamics beyond the equilibrium length scale $L^{*}$. In addition, we have prepared FL lattices in different ways to address the nonequilibrium structures. For example, we cooled the samples in the absence of a field to $65 \mathrm{~K}$, applied a field $70 \mathrm{G}$, and then cooled slowly $(0.1 \mathrm{~K} / \mathrm{min})$ to $4 \mathrm{~K}$. Significantly, we find a similar density of dislocations and FL structure compared to the rapid $(10 \mathrm{~K} / \mathrm{s})$ field-cooled samples. Since $65 \mathrm{~K}$ is far below the melting temperature [16], this observation suggests that the nonequilibrium structures originate from the frozen-in dynamics far below the melting temperature. Although we can probe FLs up to a length scale of $\sim 300 a_{0}$, there is a much smaller length scale $L^{*}$ that prohibits direct application of the theory derived for an equilibrium FLs. Further studies should address this important issue.

Finally, we consider the origin of dislocations observed in our experiments, since nonequilibrium vs. equilibrium nature of dislocations is critical to the existence of the Bragg glass phase. We believe that our data, which exhibit the small numbers of dislocations, in fact, favors nonequilibrium nature of dislocation in the FL lattice we probed by following reasons. First, it is found that most dislocations are pinned in between domain boundaries (see Fig 1(b) for example). If there were a dislocation within the domain-like structures where FLs are locally in equilibrium, the dislocation should be an equilibrium feature. Second, $L^{*} \ll L_{d}=n_{d}^{-1 / 2} \sim 250 a_{0}$, where $L_{d}$ and $n_{d}$ are the average distance between dislocations and the density of dislocations, respectively. If dislocations were energetically favorable in an equilibrium FL lattice, large dislocation loops should proliferate beyond the equilibrium length scale $L^{*}$. In addition, if some dislocations drift within domains, and are pinned at domain boundaries, we should have $L_{d} \lesssim L^{*}$. Therefore, our experiment $\left(L^{*} \ll L_{d}\right)$ suggests that dislocations are not equilibrium features in the FL lattice. Together, our data provide a lower bound for the length scale of equilibrium dislocation loop in the FL lattice.

In summary, we have obtained large scale dislocationfree images of the FL lattice in high quality BSCCO superconductors. Quantitative analyses of the translational order indicate that the system is in equilibrium for length scales up to $\sim 80 a_{0}$, and that FLs are in the random manifold regime with a roughening exponent $2 \nu=0.44$. We suggest that the very small density of dislocations observed in our data is an out-of-equilibrium feature due to the short time scales involved in our field-cooled experiments.

We thank D. R. Nelson, D. S. Fisher, P. Le Doussal, and T. Giamarchi for helpful discussion. CML acknowledges support of this work by the NSF Division of Materials Research.

* Present address: Department of Applied Physics and DIMES, Delft University of Technology, Lorentzweg 1, 2628 CJ Delft, Netherlands.

$\dagger$ Present address: Bell Laboratories, Lucent technologies, 700 Mountain Avenue, Murray Hill, NJ 07974.

[1] G. Blatter, M. V. Feigelman, V. M. Genshkenbein, A. I. Larkin, and V. M. Vinokur, Rev. Mod. Phys. 48 , 1125 (1994).

[2] A. I. Larkin, Sov. Phys. JETP 31, 784 (1970).

[3] M. V. Feigelman, V. M. Genshkenbein, A. I. Larkin, and V. M. Vinokur, Phys. Rev. Lett. 63, 2303 (1989)

[4] T. Nattermann, Phys. Rev. Lett.64, 2454 (1990).

[5] T. Giamarchi and P. Le Doussal, Phys. Rev. Lett. 72, 1530 (1994), Phys. Rev. B 52, 1242 (1995).

[6] R. Cubitt, E. M. Forgan, G. Yang, S. L. Lee, D. Mck. Paul, H. A. Mook, M. Yethiraj, P. H. Kes, T. W. Li, A. Menovsky, Z. Tarnawski, and K. Mortensen, Nature 365, 407 (1993).

[7] B. Khaykovich, E. Zeldov, D. Majer, T.W. Li, P. H. Kes, and M. Kanczylowski, Phys. Rev. Lett. 76, 2555 (1996).

[8] D. S. Fisher, Phys. Rev. Lett. 78, 1964 (1997).

[9] J. Kierfeld, T. Nattermann, and T. Hwa, Phys. Rev. B 55, 626 (1997).

[10] D. G. Grier, C. A. Murray, C. A. Bolle, P. L. Gammel, and D. J. Bishop, Phys. Rev. Lett. 66, 2270 (1991).

[11] P. Kim, Z. Yao, and C. M. Lieber, Phys. Rev. Lett. 77, 5118 (1996).

[12] Y. Li, J. Liu, and C. M. Lieber, Phys. Rev. Lett. 70, 3494 (1993).

[13] P. Kim, Ph. D. thesis, Harvard University (1999).

[14] Due to the resolution limit of ordinary printed material, we cannot present our raw data, and delaunay triangulations here. They can be found in our web site : http://magic.harvard.edu/decoration/

[15] P. M. Chaikin and T. C. Lubensky,Principles of condensed matter physics (Cambridge University Press, Cambridge, 1995).

[16] D. Majer, E. Zeldov, and M. Konczykowski, Phys. Rev. Lett. 75, 1166 (1996). 
FIG. 1. (color) Spatial map of vortex displacements $\mathbf{u}(\mathbf{r})$ from the perfect lattice positions. Darker (brighter) regions in the map correspond to smaller (larger) displacements. Different colors correspond to vortex displacements in different directions, as shown in the inserted color wheel. The two solid lines, inner and outer circles in the color wheel correspond to two basis vectors of the lattice, displacements of 0.5 $a_{0}$ and $a_{0}$ respectively. Samples were decorated at 70 G. (a) dislocation-free image containing 37003 vortices. The edge of the image correspond to $106 \mu \mathrm{m}$. The lower inset shows a part of both real FL image and a perfect lattice (yellow) with displacement vectors (red). (b) larger scale image containing three dislocations (highlighted by red dots and circles) and large scale shearing. The image contains 78385 vortices in the $160 \times 160 \mu m^{2}$ area.
FIG. 2. (a) Mean-square relative displacement correlator $B(r)$ (open circles) as a function of distance $\mathrm{r}$ calculated from the image shown in Fig 1(a). The solid line is a power law fit : $B(r) \sim r^{0.44}$.(b) Translational correlation function $C_{\mathbf{G}}(r)$ (dots) calculated from the same image. The open circles are a comparison with the Gaussian approximation : $C_{\mathbf{G}}(r) \approx e^{-G^{2} B(r) / 2}$

FIG. 3. (a) Transverse (open circle) and longitudinal (solid circle) displacement correlators, i.e. $B^{T}(r)$ and $B^{L}(r)$, as a function of distance calculated from Fig. 11(a). The insert shows the ration of the two quantities. (b) The Fourier space image showing all six first order Bragg peaks. (c) Detailed of one of the Fourier peaks calculated from the same image (inverted gray scale). Dark arrows highlight two satellite peaks. 
This figure "Fig1.GIF" is available in "GIF" format from: http://arxiv.org/ps/cond-mat/9910184v2 
This figure "Fig2.GIF" is available in "GIF" format from: http://arxiv.org/ps/cond-mat/9910184v2 
This figure "Fig3.GIF" is available in "GIF" format from: http://arxiv.org/ps/cond-mat/9910184v2 